\title{
Diferentes técnicas de integración paisajística en carreteras. Análisis de eficacia a través de la percepción de observadores
}

\section{Different landscaping integration techniques in roads. Analysis of efficacy through public perception}

B. Martín $^{(*)}$, M. Loro(*), R. M. Arce $e^{(*)}$, I. Otero(*)

RESUMEN

En el trabajo que se expone en este artículo se planteó como objetivo realizar un análisis de las diferentes medidas de integración paisajística que se utilizan en las autopistas y autovías españolas. Para ello, se ha realizado una revisión bibliográfica de la normativa, la documentación científica, las guías y demás recomendaciones en este ámbito, y se ha efectuado un inventario fotográfico que recoge las medidas de restauración observadas en las diferentes vías. Gracias a una encuesta fotográfica y al posterior análisis de la percepción del público de las diferentes medidas de integración, se han identificado las variables medioambientales clave en la integración de las autopistas en el paisaje, los aspectos de diseño más adecuados y las medidas de integración paisajística más efectivas.

\section{SUMMARY}

In the present work the objective is to analyze the landscaping integration measures commonly used in Spanish highways. In order to achieve this objective a review of main normative, scientific documentation, guidelines and handbooks in this framework was carried out. Furthermore, a photographic inventory and a survey aimed at knowing the public perception of different road landscaping techniques were done. The survey results analysis allowed us to identify key environmental variables in highway landscaping, adequate design techniques and the efficacy of the landscaping measures studied.
Palabras clave: Paisaje; preferencias; público; integración; autovías/autopistas.
Keywords: Landscape; preferences; public; integration; highways.
(*) TRANSyT - Universidad Politécnica de Madrid. Madrid (España) Persona de contacto/Corresponding author: bmartin@caminos.upm.es (B. Martín) 


\section{INTRODUCCIÓN}

Las carreteras no solo son vías de transporte, sino que constituyen un elemento estructural del paisaje (1) y un medio a través del cual el individuo entra en contacto con el mismo (2) (3). Así, las carreteras pueden suponer un impacto negativo de antropización y otro positivo de comunicación con el paisaje.

Generalmente, los estudios de impactos se centran en la alteración introducida por la infraestructura vista desde fuera, pero el conductor/observador que circula por la misma no solo ve el paisaje exterior. El paisaje más próximo está muy presente a lo largo de su viaje y la presencia de alteraciones asociadas a la carretera influye en la escena observada. Por tanto, a la hora de evaluar impactos y establecer medidas correctoras, las características visuales de las áreas alteradas percibidas desde la propia vía deben ser tenidas igualmente en cuenta (3)(4).

Para estimar qué medidas correctoras deben aplicarse es necesario valorar el cambio originado en la calidad visual del lugar donde se localiza el proyecto y estimar en qué grado se recupera la calidad perdida gracias a ellas. Tal cambio dependerá tanto de la calidad intrínseca de la zona afectada como de su fragilidad visual, característica fundamental para el diseño de medidas correctoras (3).

A la hora de determinar qué tipo de medida correctora puede aplicarse, generalmente se recurre a las recomendaciones de guías y manuales técnicos, pero en éstas se suelen dar recomendaciones generales, sin especificar en qué grado se mejorará el paisaje aplicando dicha medida.

En el trabajo que se expone en el presente artículo se planteó como objetivo último definir qué se considera una buena integración de la carretera en el paisaje y cuantificar las mejoras conseguidas. Para estimar dicha efectividad, se ha realizado una encuesta para estimar cómo varía la percepción del público ante diferentes soluciones constructivas y diseños (5). Para la creación de escenarios de evaluación se ha recurrido a simulaciones fotográficas de medidas correctoras y otros elementos de la carretera. Este tipo de técnicas son frecuentemente utilizadas en el período de información pública del Estudio Informativo (3).

La labor de integración paisajística de una autopista o una autovía no acaba en la fase de construcción de la infraestructura puesto que, en muchas ocasiones, las concesionarias encargadas de su gestión y mantenimiento mejoran dicha integración (6). Fruto del interés por mejorar su labor de integración paisajística, nace el proyecto OASIS (Operación de Autopistas Seguras Inteligentes y Sostenibles).

Los resultados que se exponen en el presente artículo son fruto de los trabajos de investigación realizados en el marco del Proyecto OASIS, subvencionado por el Centro para el Desarrollo Tecnológico e Industrial (CDTI) dentro del programa CENIT. Dichos resultados son, pues, propiedad exclusiva de las empresas que promueven dicho proyecto y que constituyen la Agrupación de Interés Económico OASIS-CENIT, A.I.E. (OHL-Concesiones, ABERTIS, IRIDIUM, INDRA, SICE, OHL, DRAGADOS, GMV y GEOCISA).

\subsection{La importancia del paisaje interior de la carretera}

Las autopistas generan una topografía propia que se superpone al territorio que atraviesan y, en ocasiones, esta nueva topografía puede generar un impacto en el paisaje importante. Para minimizar ese impacto y permitir que la autopista sea lo más compatible posible con el territorio, existen una serie de elementos del medio que han de considerarse en su diseño. Algunas de estas consideraciones son:

- El propio paisaje como condicionante del diseño del trazado.

- La vegetación como elemento integrador, pero también con funciones en la seguridad vial y de ecología del paisaje.

- La elección de diseños constructivos acordes con los atributos del paisaje: formas, colores y texturas.

Dado que la investigación realizada está orientada a la mejora del paisaje interior de la carretera, es decir, la percepción que tiene el observador de los elementos propios de la infraestructura y del paisaje que se divisa desde ella (7), se ha estudiado cómo la modificación de dichos elementos puede cambiar la percepción del paisaje que tiene el usuario de la carretera.

\subsection{Identificación de los elementos influyentes en integración paisajística de las autopistas}

De forma general pueden plantearse dos grupos de medidas orientadas a la mejora del paisaje interior. Un grupo englobaría aquellas medidas orientadas a la búsqueda de la naturalización del paisaje con nuevas plantaciones y con la corrección de la topografía alterada. El otro grupo estaría enfocado a la búsqueda de elementos constructivos con un carácter más integrador (ver Tabla 1). 
Tabla 1. Relación de medidas de integración paisajística más utilizadas

\begin{tabular}{|c|c|}
\hline \multicolumn{2}{|c|}{ Mejora en la integración del paisaje: plantaciones y corrección de la topografía } \\
\hline \multirow{2}{*}{ Taludes } & Corrección geométrica de desmontes y terraplenes, adaptándose a la topografía \\
\hline & Tratamientos de revegetación de taludes \\
\hline \multirow{3}{*}{ Túnel } & Tratamiento de la boca del túnel y diseño de la bóveda adaptándola a la ladera \\
\hline & Utilización de colores miméticos con el paisaje \\
\hline & Tratamiento de revegetación en la boca del túnel \\
\hline \multirow{3}{*}{ Plantaciones } & Ocultación de elementos que alteran el paisaje: pantallas vegetales \\
\hline & Realce de las características paisajísticas de la carretera \\
\hline & Orientación visual del trazado y reducción del deslumbramiento \\
\hline \multicolumn{2}{|r|}{ Elementos constructivos con un carácter más integrador } \\
\hline \multirow{2}{*}{ Muros de contención } & Muros de hormigón con diferentes tramas \\
\hline & Muros de materiales "naturales": gaviones, escolleras, evergreen, etc. \\
\hline \multirow{3}{*}{ Pantallas acústicas } & Pantallas prefabricadas: transparentes, opacas, prefabricadas y motas de tierra \\
\hline & Utilización de colores miméticos con el paisaje \\
\hline & Pantallas acústicas acompañadas por plantaciones \\
\hline \multirow{4}{*}{ Pasos superiores } & Utilización de colores miméticos \\
\hline & Diseños ligeros, con pilares y vigas que tapen poco las vistas \\
\hline & Incorporación de la vegetación para mejorar la calidad estética del mismo \\
\hline & Búsqueda de diseños con formas similares a las formas predominantes de la zona \\
\hline
\end{tabular}

\subsection{Estudios de opinión en el diseño de infraestructuras o en la integración paisajística}

Las guías y manuales técnicos relacionados con la integración paisajística de infraestructuras lineales, generalmente se centran en aspectos relativos a la lectura geométrica de la carretera, más que en los valores estéticos y paisajísticos del territorio a preservar (8). La complejidad técnica que existe para definir qué valores estéticos son más importantes y qué medidas son más eficaces en la reducción del impacto paisajístico hace que las encuestas de opinión sean un apoyo para identificar y medir los valores estéticos, culturales o sociales que merecen ser preservados (8).

En ocasiones, las encuestas de percepción del paisaje se incorporan en procesos de participación ciudadana. Estos procesos son impuestos por algunas legislaciones autonómicas (9) (10), pero generalmente se centran en planes y programas de carácter territorial y ambiental, pero no en escala proyecto (11).

A raíz de la detección de dicha deficiencia, se ha considerado interesante realizar una encuesta centrada en evaluar de qué manera se aproximan las valoraciones más altas de la calidad del paisaje a la aplicación de determinadas medidas en una escala de mayor detalle.

\section{MATERIAL Y MÉTODOS}

\subsection{Diseño de la encuesta y método de análisis}

Para conocer mejor la preferencia de los observadores en cuanto a las medidas de integración anteriormente descritas, se diseñó una encuesta que constaba de 5 cuestionarios que contenían fotografías de paisajes. En los estudios de percepción de paisaje es común el uso de fotografías como alternativa a la observación directa del paisaje. Diversas investigaciones han demostrado que el uso de fotos no plantea problemas cuando se trata de recoger las reacciones del público ante escenas de localizaciones exteriores $(12,13,14)$.

Cada cuestionario constaba de dos módulos, uno con 9 preguntas sobre las características socio-económicas y otros aspectos que pudieran ser interesantes para evaluar diferencias entre grupos de encuestados y otro módulo con 25 o 26 fotografías de paisajes y de medidas de integración en autopistas. El análisis de los grupos de encuestados no se discutirá en este artículo.

La mayor parte de las fotografías incluidas en los cuestionarios fueron tomadas en un inventario de los paisajes observados desde autopistas españolas seleccionadas. Las carreteras que se recorrieron y fotografiaron durante el inventario fotográfico se representan en la Figura 1. 
De entre el conjunto de fotografías tomadas, se seleccionaron aquellas que mostraban la aplicación de medidas de integración en carreteras. Las fotos seleccionadas fueron reveladas en color, con una resolución de 254 ppp y unas dimensiones de 18 x 24 cm (2400 x 1800 píxeles).

En total, se seleccionaron 128 fotografías para la encuesta, de las cuales 79 correspondían a medidas de integración paisajística y 49 a paisajes clasificados en 7 calidades (degradado, deficiente, mediocre, bueno, notable, muy bueno, excelente). Para esta clasificación se utilizó un método de valoración basado en la posesión de atributos (19). Este método ha sido utilizado con éxito en diferentes trabajos de investigación sobre evaluación del paisaje $(1,20$, 21,22 ). De las 73 fotografías destinadas a conocer la mejora del paisaje al incluir medidas de integración, algunas fueron modificadas utilizando el software de tratamiento fotográfico Adobe Photoshop CS2. Las modificaciones simulaban medidas correctoras encaminadas a la reducción del impacto introducido por la infraestructura en el paisaje. Las 128 fotografías se dividieron en 5 grupos (series o cuestionarios) homogéneos en cuanto a tipos de paisaje y medidas de integración, procurando que no hubiera en el mismo cuestionario varias fotografías modificadas a partir de la misma foto original y que todas los tipos de calidad del paisaje estuvieran representados. Estas fotos se valoraron por el público encuestado en una escala del 1 al 5 ( 1 = muy malo; $5=$ muy bueno). Esta escala de valoración se ha seleccionado en base a estudios previos de características similares $(15,16,17,18)$.

En la Tabla 2 se muestra la distribución de las fotografías en los diferentes tipos de cuestionarios. Una vez seleccionadas las fotografías que constituirían cada serie, se ordenaron de manera aleatoria en el cuestionario.

Tabla 2. Distribución de las fotografías en los cuestionarios.

\begin{tabular}{|c|c|c|c|c|c|c|c|}
\hline & TIPO DE FOTO & $\begin{array}{c}\text { SERIE } 1 \\
n^{\circ} \text { fotos }\end{array}$ & $\begin{array}{l}\text { SERIE } 2 \\
n^{\circ} \text { fotos }\end{array}$ & $\begin{array}{c}\text { SERIE } 3 \\
n^{\circ} \text { fotos }\end{array}$ & $\begin{array}{c}\text { SERIE } 4 \\
n^{\circ} \text { fotos }\end{array}$ & $\begin{array}{c}\text { SERIE } 5 \\
n^{\circ} \text { fotos }\end{array}$ & TOTAL \\
\hline \multirow{8}{*}{$\begin{array}{c}M \\
E \\
D \\
\text { I } \\
D \\
\text { A } \\
\text { S }\end{array}$} & Pantallas acústicas & 1 & 1 & 2 & 1 & 2 & 7 \\
\hline & Medianas & 2 & 1 & 1 & 1 & 1 & 6 \\
\hline & Taludes & 2 & 2 & 2 & 1 & 1 & 8 \\
\hline & Pantallas vegetales & 2 & 1 & 1 & 2 & 1 & 7 \\
\hline & Pasos superiores & 3 & 3 & 3 & 3 & 4 & 16 \\
\hline & Túneles & 2 & 3 & 3 & 5 & 3 & 16 \\
\hline & Pasarelas & 2 & 3 & 4 & 3 & 3 & 15 \\
\hline & Muros de contención & 1 & 1 & 1 & 1 & 1 & 5 \\
\hline \multicolumn{2}{|c|}{ FOTOS DE PAISAJES } & 11 & 11 & 9 & 9 & 9 & 49 \\
\hline \multicolumn{2}{|c|}{ TOTAL } & 26 & 26 & 26 & 25 & 25 & 128 \\
\hline
\end{tabular}

Las encuestas se realizaron mediante dos canales. En primer lugar, se hizo una campaña de encuestas en 5 áreas de servicio y descanso de autopistas y, posteriormente, la misma encuesta se lanzó a través de una página web.

El tamaño de muestra inicial estimado (n_0 en [1]) fue de 89 personas. Para obtenerlo se usó la técnica de muestreo aleatorio simple, utilizando la desviación típica obtenida en un estudio previo similar (16):

$$
n_{0}=\left(\frac{\sigma \cdot z_{(1-\alpha / 2)}}{d}\right)^{2}
$$

Donde:

$n_{0}$ es el tamaño de la muestra.

$\sigma$ es la desviación típica de la distribución, el valor de $\sigma$ escogido para estimar la muestra fue 0,48 (16).

$d$ es el error de muestreo, en este caso se fijó un error 0,1 .

$Z_{(1-\alpha / 2)}$ es el valor de la variable normal estándar (1,98 con un nivel de confianza $\alpha=95 \%$ ).

El número total de respuestas por cada serie de fotografías fue de 737 respuestas (Tabla 3).

Tabla 3. Número de encuestados.

\begin{tabular}{lccccc} 
& $\begin{array}{c}\text { Serie } \\
\mathbf{1}\end{array}$ & $\begin{array}{c}\text { Serie } \\
\mathbf{2}\end{array}$ & $\begin{array}{c}\text { Serie } \\
\mathbf{3}\end{array}$ & $\begin{array}{c}\text { Serie } \\
\mathbf{4}\end{array}$ & $\begin{array}{c}\text { Serie } \\
\mathbf{5}\end{array}$ \\
\hline $\begin{array}{l}\text { Número de } \\
\text { encuestados }\end{array}$ & 145 & 148 & 146 & 150 & 148 \\
\hline
\end{tabular}

Para analizar si las mejoras en la integración paisajística introducidas en las fotografías se traducen en una mejora de la calidad del paisaje, se estudiaron las medias, desviaciones típicas y varianzas de las puntuaciones que los encuestados otorgaron a cada fotografía.

La igualdad entre las medias se analizó utilizando análisis de la varianza (ADEVA, $\alpha=95 \%$ ) y el test de los múltiples rangos (método Duncan, $\alpha=95 \%$ ) en todos los grupos de fotografías (formados por la foto original y los fotomontajes creados a partir de la misma), y el test de la T de Student $(\alpha=95 \%)$ cuando se trataba de comparar dos fotografías. La hipótesis nula de los test es que las medias son iguales, es decir, que los encuestados no notan mejora en la calidad del paisaje entre la foto original y la retocada o retocadas (caso de que fuera más de una). En cambio, la hipótesis alternativa considera que la media de las valoraciones de las fotos es mayor cuando se introducen mejoras en el paisaje.

La igualdad de las medianas se estudió planteando las mismas hipótesis de igualdad, utilizando los test de Wilcoxon, en el caso de dos muestras (original y su modificada), y el de Kruskal Wallis ( $\alpha=95 \%$ ) en los casos en los que se analizaron más de dos fotografías (original y varias modificadas, a partir de esa foto original).

A los resultados obtenidos en la encuesta también se les ha aplicado el test de Cochran $(\alpha=95 \%)$ con el objetivo de confirmar 
la igualdad en las desviaciones típicas de todas las muestras obtenidas. La igualdad entre las desviaciones típicas indica que la dispersión de los datos es similar en las muestras, lo cual indicaría que los encuestados discriminan de forma similar entre las diferentes fotografías.

Dado el nivel de confianza $\alpha$ establecido, si el p-valor que se obtiene en los test planteados es mayor que 0,05 , se acepta la hipótesis nula, en caso contrario, se rechaza a favor de la alternativa. Todos los análisis fueron realizados utilizando el software Statgraphics Centurion VI.

\subsection{Elementos influyentes en integración paisajística de las autopistas analizadas}

En este estudio se ha hecho una aproximación utilizando un número muy limitado de fotografías, dando una idea de la variación en la percepción del público de la calidad de un paisaje, en función de la aplicación de diferentes medidas.

Se han llamado escenarios al conjunto que componen una fotografía y las modificaciones de la misma. A continuación, se resumen los elementos estudiados y los diferentes escenarios planteados.

\subsubsection{Túneles}

Cuando se diseña la restauración paisajística de una entrada de túnel se recomienda tener en cuenta tanto el acabado del emboquillado, como la revegetación de todos los elementos que rodean a la embocadura. Por ello, se incluyeron los siguientes escenarios en la encuesta:

1. Entrada de túnel en un entorno boscoso: se introducen elementos de colores miméticos como medida de integración del emboquillado (Figura 2).

2. Túnel en un entorno boscoso en el que se evalúa la tipología de plantación realizada (hidrosiembra y arbolado) (Figura 3).

3. Entrada de túnel en una zona mediterránea caracterizada por la presencia de abundante matorral, en el que se ha tenido que aplicar un gunitado para estabilizar el talud (Figura 4).

4. Túnel en un entorno muy rocoso, donde es perceptible el corte realizado a la roca durante la construcción del túnel. En la foto modificada se ha avanzado la boca del túnel para restituir el talud original y se han introducido plantaciones (Figura 5).

5. Entrada de túnel con diferentes diseños constructivos de emboquillado orientados a "naturalizar" la misma. Para ello, se reduce el grosor del marco prefabricado con diseños sencillos. Además, se

ha evaluado otro tipo de soluciones que buscan mostrar el túnel como elemento a destacar, efecto conseguido con la incorporación de elementos decorativos (marcos temáticos, insignias) o con encachados y muros prefabricados (algunas fotos se muestran en la Figura 6).
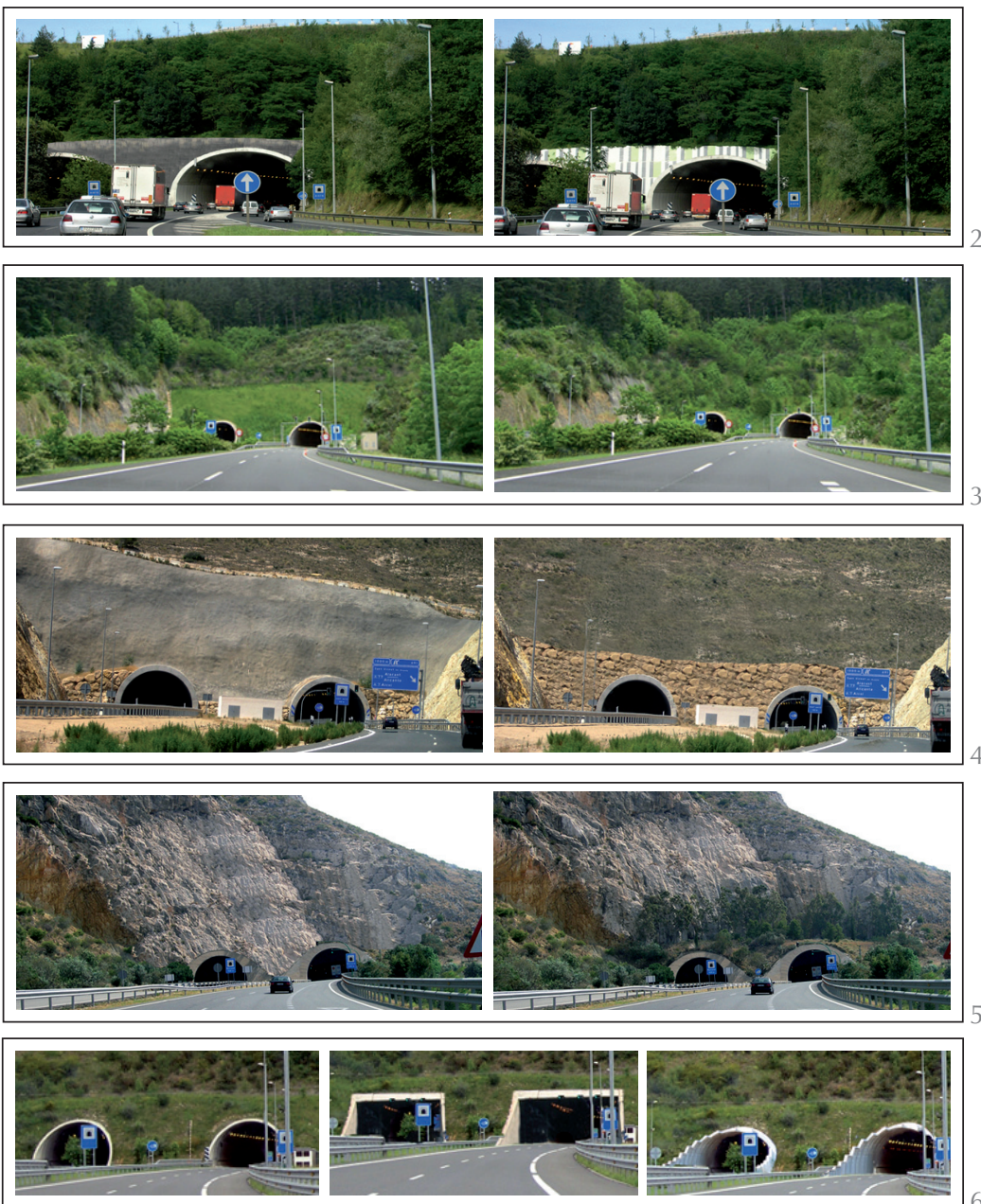

2.2.2. Eficacia en el uso de las pantallas vegetales

Los principales parámetros que condicionan el éxito de la aplicación de esta medida son: la calidad paisajística de la zona donde se proyecta la pantalla, las características de elemento a ocultar y la diversidad de especies vegetales que conforman la pantalla. Para analizar estos aspectos, se han creado tres escenarios de estudio:

1. Visión de un polígono industrial desde la autopista, en una zona con destacado interés paisajístico. Para mejorar el paisaje se ha diseñado una pantalla vegetal formada por diversas especies vegetales similares a las presentes en la zona (Figura 7).

2. Visión de una zona residencial desde la autopista, en una zona con un interés paisajístico medio. Para mejorar el paisaje se ha diseñado una pantalla vegetal formada por pinos (Figura 8).
2. Escenario 1: integración de túnel con colores miméticos (fotos S45 y S53).

3. Escenario 2: integración de boca de túnel mediante hidrosiembras y plantaciones (código encuesta S217 y S115).

4. Escenario 3: túnel con taludes gunitados (código S422 y S521).

5. Escenario 4 túnel con pronunciado corte en la roca (códigos S48 y S319)

6. Escenario 5: comparación de emboquillados de túnel. Fotos S510, S323 y S321 (de izq. a der.). 
3. Visión de una zona industrial desde la autopista, en una zona con interés paisajístico medio a medio bajo. Para mejorar el paisaje de esta zona se ha diseñado una pantalla vegetal formada por un seto (Figura 9).
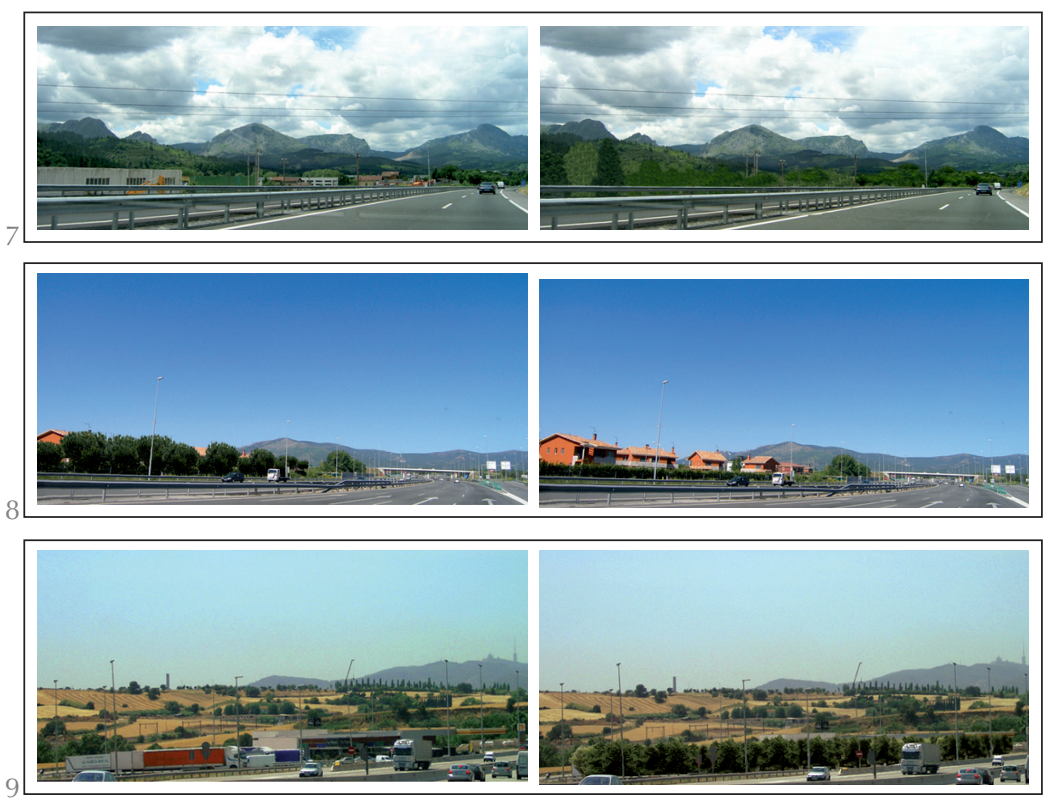

7. Escenario 1 pantallas vegetales (fotos S325 y S112).

8. Escenario 2 pantallas vegetales (fotos S118 y S29).

9. Escenario 3 pantallas vegetales (fotos S410 y S513).

10. Escenario 1 taludes: plantaciones en taludes de grandes dimensiones (S111 y S220).

11. Escenario 2 taludes: Integración de taludes rocosos (fotos S55 y S28).

\section{Escenario taludes: tratamien-} tos en tramo en trinchera (fotos S315 y S417).

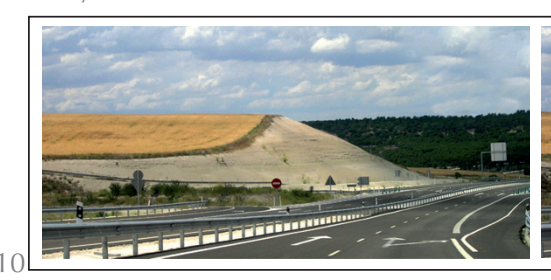

\subsubsection{Taludes}

Para realizar el estudio sobre la integración paisajística de taludes se han seleccionado los siguientes escenarios:

1. Grandes taludes donde se diseña una plantación de árboles en banquetas (Figura 10).

2. Taludes de roca con fuerte inclinación en los que solo se pueden extender geotextiles y mantas orgánicas para mejorar su integración (Figura 11).

3. Taludes de escasa altura, pero con una fuerte inclinación en un tramo en trinchera, en los que se introduce vegetación (Figura 12).
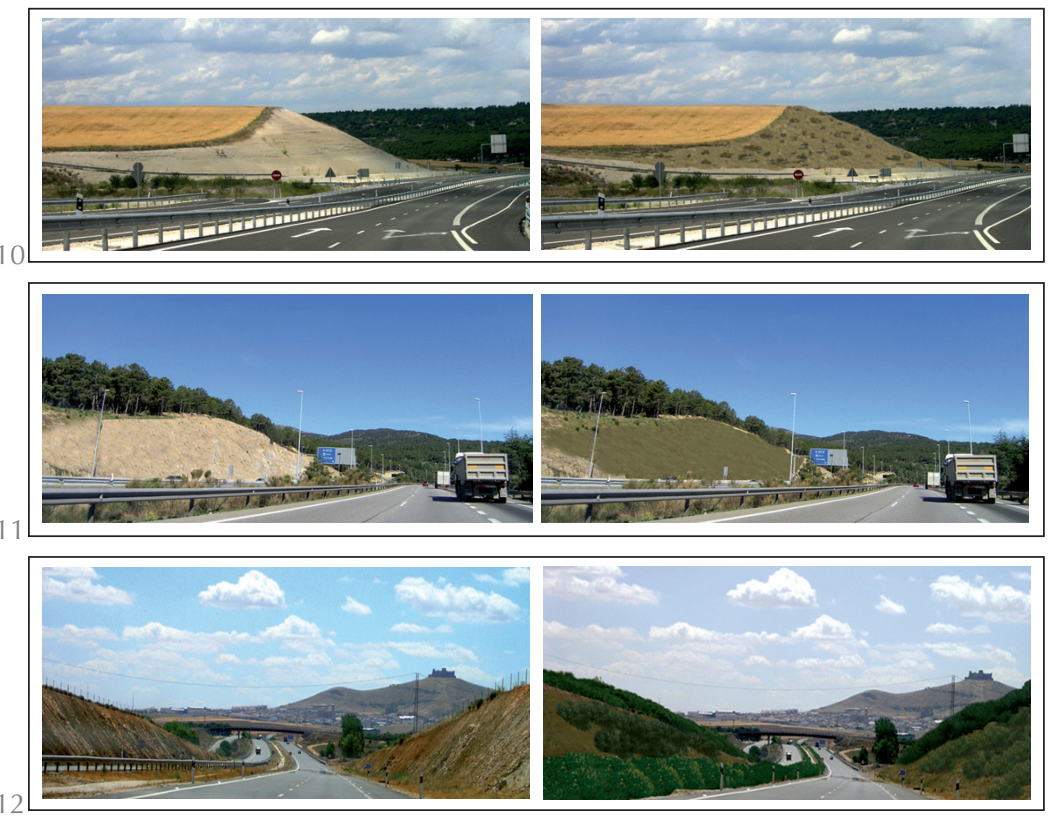

\subsubsection{Pasos superiores}

El estudio de la integración paisajística de pasos superiores se ha centrado en dos aspectos. Por un lado, se han estudiado diferentes escenarios donde se han aplicado plantaciones en taludes y cambios de color de algunos elementos estructurales $y$, por otro, se han seleccionado 2 escenarios donde se han modificado elementos estructurales del paso superior, variando así la ligereza del mismo:

1. Paso superior localizado en un paisaje con olivares típicamente mediterráneo, próximo a una zona con taludes rocosos en trinchera. Se restauran los taludes próximos al paso superior (Figura 13).

2. Paso superior con taludes desnudos, próximo a una zona de pinares y de pequeñas montañas. Se introduce vegetación y cambios en el color de la barandilla para evaluar las preferencias del público (Figura 14).

3. Paso superior sustituido por otro más ligero y adaptado a las formas del terreno, permitiendo apreciar con mayor nitidez el paisaje de la zona (Figura 15).

4. Paso superior localizado en la España mediterránea, que es visto desde un tramo de autopista en trinchera y con un punto de interés al fondo de la escena. Dadas las características del tramo, la atención del observador se dirige al fondo de la escena. Se busca conocer si existen diferencias en la preferencia del observador, variando el diseño de los diferentes elementos constructivos del paso superior, como son los estribos o los pilares (Figura 16).

\subsubsection{Muros de contención de tierras}

Se ha seleccionado un escenario base para estudiar cómo la propuesta de diferentes tipologías de muros de contención de tierras puede variar la calidad estética de un paisaje. El escenario elegido se caracteriza por su alto valor estético, con bosques caducifolios compuestos por diversas especies, presencia de altas montañas al fondo de la escena y la ausencia de elementos que alteren la vista, a excepción del muro evaluado. Se ha seleccionado este escenario, con un nivel inicial de calidad paisajística buena, para evaluar cómo varía dicha calidad al modificar el muro de contención, procurando seleccionar diseños que lo hagan más perceptible al observador. Las tipologías de muros seleccionados son las siguientes (Figura 17):

- Muro de gaviones

- Muro de escamas prefabricadas de hormigón

- Muro de pantalla prefabricada.

- Muro de lamas con jardineras.

- Muro prefabricado con elementos de hormigón con un acabado de cara rústica. 

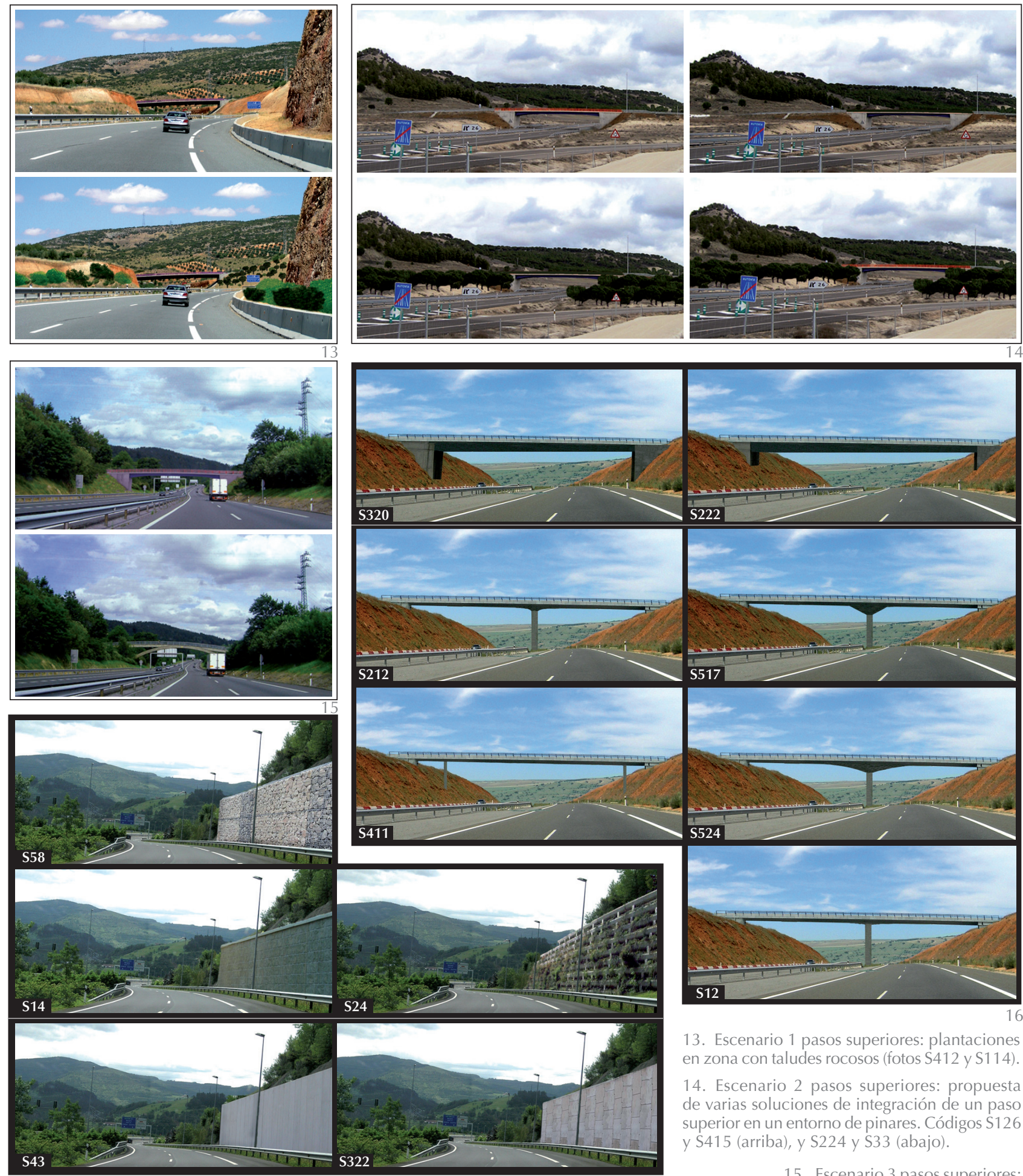

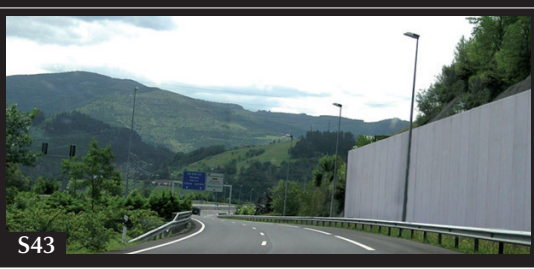

2.2.6. Pantallas acústicas

El estudio de la integración paisajística de las pantallas acústicas se ha centrado en evaluar, por un lado, la mejora del paisaje con la creación de pantallas vegetales que las oculten al observador y, por otro lado, qué tipología de pantalla acústica resulta más adecuada cuando se pretende buscar una mejor integración en el paisaje. Para evaluar estos criterios se han creado 3 escenarios de estudio. Las características principales de estos escenarios son las siguientes:
5. Paisaje con alto valor paisajístico en el que aparece una pantalla acústica como elemento de alteración. Se ha creado una pantalla vegetal con la intención de evaluar si mejora el paisaje al ocultar la alteración (Figura 18)

6. Pantalla acústica en un paisaje de calidad media donde se ha modificado la tipología de pantalla acústica (Figura 19).

7. Evaluación de la efectividad paisajística de pantallas acústicas opacas frente al uso de pantallas transparentes (Figura 20).
13. Escenario 1 pasos superiores: plantaciones

Escenario 2 pasos superiores: propuesta varias soluciones de integración de un paso uperior en un entorno de pinares. Códigos S126 S415 (arriba), y S224 y S33 (abajo).

15. Escenario 3 pasos superiores: estudio de tipologías constructivas de pasos superiores (código S518 y S37)

16. Escenario 4 pasos superiores: Pasos superiores con diseños poco aligerados (foto S320 y S222). Pasos superiores con diseños muy aligerados. Códigos encuesta S212, S517, S411, S524 y S12.

17. Escenario 1 muros: Aplicacion de muros de contención con acabados más naturales, código encuesta S58, S14 y S24. Aplicación de muros de contención menos integradores, código S43 y S322. 

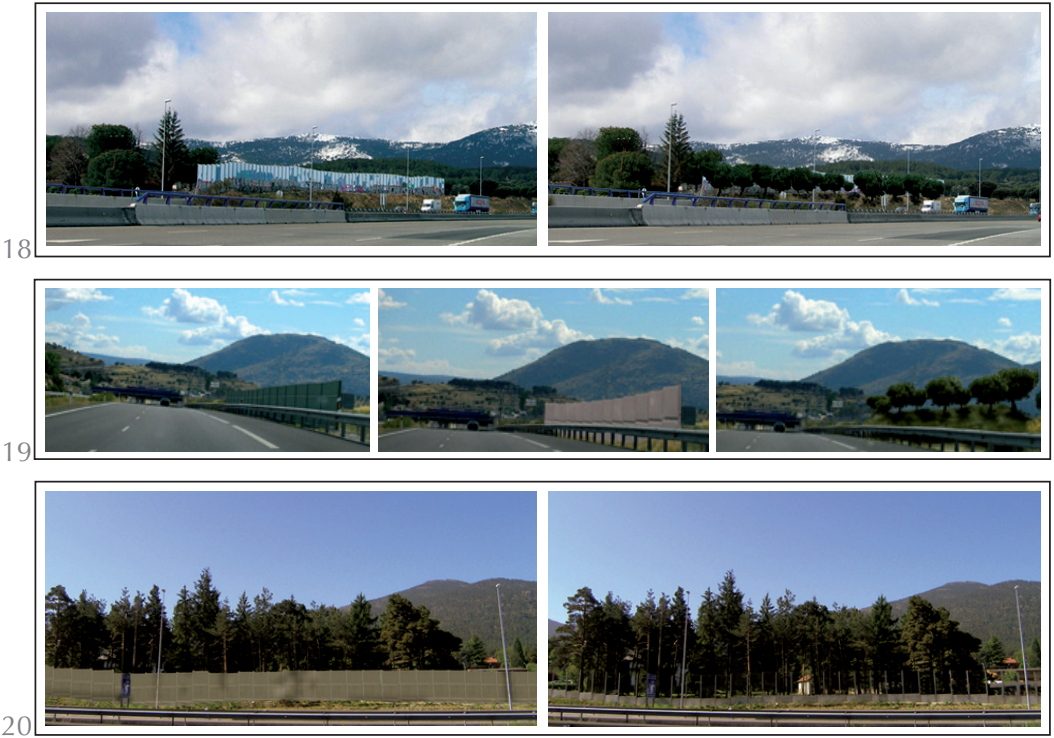

2.2.7. Medianas

18. Escenario 1 pantallas acústicas: ocultación de pantallas acústicas con pantallas vegetales. Fotos S117 y S27.

19. Escenario 2 pantallas acústicas: propuesta de diversas soluciones de pantalla acústica. Fotos S312, S49 y S520 (de izq. a der.).

20. Escenario 3 pantallas acústicas: pantallas acústicas transparentes y opacas. Fotos S515 y S317.

21. Escenario 1 medianas: plantaciones en la mediana. Fotos S120 y S215.

22. Escenario 2 medianas: modificación del tipo de plantación en la mediana. Fotos S52 y S122.

23. Escenario 3 medianas: plantación en mediana en zona con amplias vistas. Fotos S34 y S46.

Las plantaciones en medianas, además de aportar calidad a la escena interior de la carretera, mejoran la legibilidad del trazado, evitan deslumbramientos con la circulación en sentido opuesto y mejoran la sensación de confort del conductor durante la conducción. Para conocer mejor cómo varía la percepción de los observadores de la autopista al introducir una mediana se han incluido en la encuesta los siguientes escenarios:

1. Tramo de autopista en curva, donde la creación de una mediana recortada ayuda a "leer" la curva y mejora la integración paisajística de la carretera (Figura 21).

2. Tramo de autopista donde se han sustituido las plantaciones de retama de bolas por adelfas (inclusión de color en el paisaje). El paisaje del tramo tiene una calidad paisajística media-baja (Figura 22).
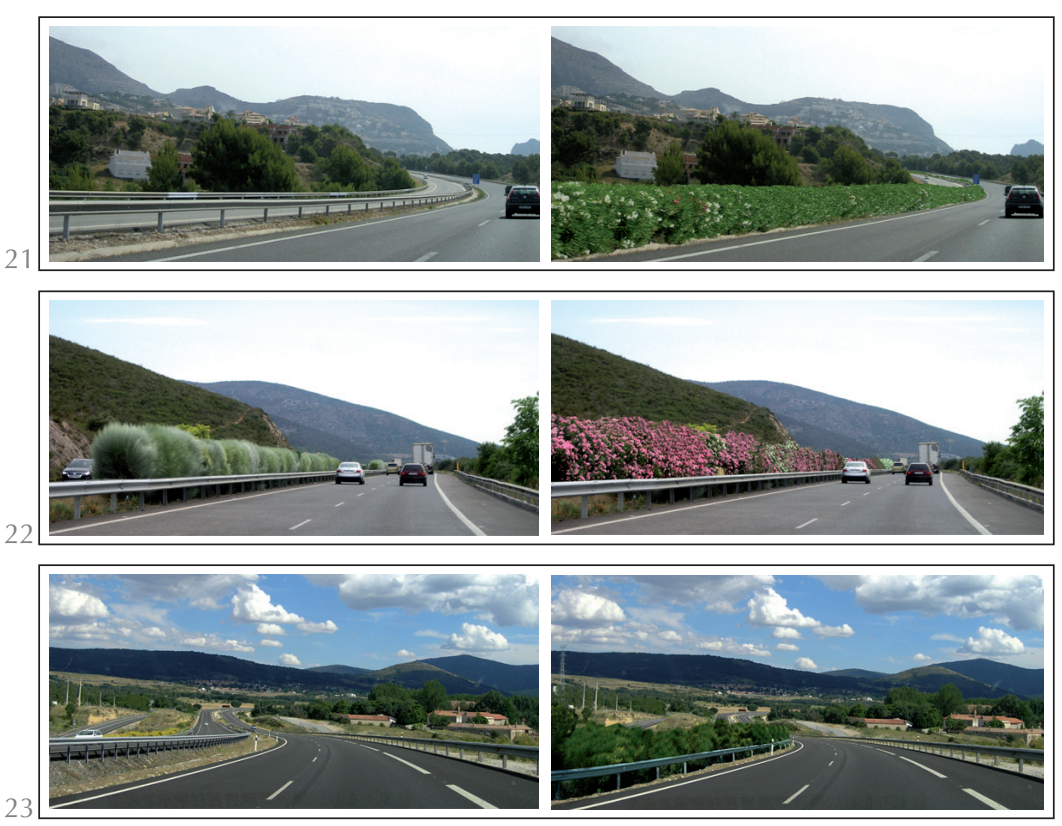

3. Plantación de la mediana en una zona con amplias vistas (Figura 23).

\section{RESULTADOS}

\subsection{Análisis de la valoración obtenida en las fotografías}

La valoración obtenida por cada fotografía se analizó a través de la valoración media de todos los encuestados para dicha foto. La Tabla 4 presenta las medias y las desviaciones típicas de cada una de las series y la total. El número de fotos utilizado (128) garantiza un error relativo $(3,92 \%)$ en la estimación de la preferencia (error absoluto \pm 0.12 ). Las medias de la valoración de las fotos presentes en las series sigue una distribución normal, no identificándose diferencias entre las medias y desviaciones típicas de las diferentes series de fotografías. Para identificar las posibles diferencias entre medias se utilizó ADEVA, y el test de Cochran para identificar diferencias entre las varianzas. Ambos valores son mayores que 0,05, por lo que se acepta la hipótesis nula de igualdad de medias y de varianzas con un $95 \%$ de confianza (Tabla 5).

\subsection{Análisis de las medidas de integración paisajística}

A continuación se presentan los resultados obtenidos en los test estadísticos paramétricos y no paramétricos realizados, así como las diferencias en las preferencias mostradas por los encuestados en las fotografías donde se modificaron elementos propios del paisaje interior de la carretera:

\subsubsection{Túneles}

En la Tabla 6 se muestran los resultados de los test estadísticos empleados En todos los escenarios, excepto en el 3, se identificaron diferencias significativas entre las medias y medianas de las valoraciones y homogeneidad en las desviaciones típicas. En el escenario 3, las desviaciones típicas son significativamente distintas al 95\% de confianza.

En los escenarios de estudio 1 y 3 se ha obtenido una mejora destacada al reducir la alteración introducida por el gunitado con la utilización de colores miméticos. Dicha mejora ha sido entre un 25 y un $30 \%$ respecto a la situación original (situación antes de aplicar la medida). Estas diferencias en porcentajes, y todas las que se incluyan de ahora en adelante, corresponden a la diferencia relativa, en tanto por ciento, entre la media de la situación original y la final, transformando la escala original (1-5) en una escala de 0 a 100. 
Tabla 4.

Medias y desviaciones típicas de las valoraciones medias de las fotografías en diferentes series y considerando todas juntas

\begin{tabular}{lcccccc}
\hline Estadístico & SERIE 1 & SERIE 2 & SERIE 3 & SERIE 4 & SERIE 5 & TOTAL \\
\hline$N^{\mathbf{0}}$ de fotos & 26 & 26 & 26 & 25 & 25 & 128 \\
\hline media & 3,06 & 3,21 & 0,16 & 3,03 & 3,17 & 3,13 \\
\hline Desviación estándar & 0,85 & 0,68 & 0,64 & 0,80 & 0,72 & 0,72 \\
\hline
\end{tabular}

Tabla 5.

Diferencias entre las medias y desviaciones típicas de las valoraciones medias en las diferentes series

\begin{tabular}{cccc}
\hline & ADEVA & \multicolumn{2}{c}{ C de Cochran } \\
\hline Razón F & Valor-p & Prueba & Valor-p \\
\hline 0,26 & 0,9026 & 0,243255 & 0,963499 \\
\hline
\end{tabular}

Tabla 6. Resultados en las fotografías con túneles

\begin{tabular}{|c|c|c|c|c|c|c|c|c|}
\hline Escenario & Fotos & media & $\begin{array}{l}\text { ADEVA } \\
\text { p-valor }\end{array}$ & $\begin{array}{l}\text { Test T Student } \\
\text { p-valor }\end{array}$ & $\begin{array}{c}\text { Test medianas } \\
\text { p-valor }\end{array}$ & $\begin{array}{l}\text { Test Cochran } \\
\text { p-valor }\end{array}$ & Test múltiples rangos & $\begin{array}{c}\text { Diferencia } \\
\text { entre medias } \\
(\%)\end{array}$ \\
\hline Escenario. 1 & $\begin{array}{l}\text { S45 } \\
\text { S53 }\end{array}$ & $\begin{array}{l}2,7909 \\
3,2517\end{array}$ & 0,0001 & 0,0001 & 0,0001 & 0,7915 & * & 25,73 \\
\hline Escenario. 2 & $\begin{array}{l}\text { S115 } \\
\text { S217 }\end{array}$ & $\begin{array}{l}3,5395 \\
4,0265\end{array}$ & 0,000 & 0,000 & 0,000 & 0,0427 & * & 19,18 \\
\hline Escenario 3 & $\begin{array}{c}\mathrm{S} 48 \\
\mathrm{~S} 319\end{array}$ & $\begin{array}{l}3,3243 \\
3,4379\end{array}$ & 0,3113 & 0,8444 & 0,8626 & 0,7154 & & \\
\hline Escenario 4 & $\begin{array}{l}\text { S422 } \\
\text { S521 }\end{array}$ & $\begin{array}{l}2,2895 \\
2,6779\end{array}$ & 0,0003 & 0,0002 & 0,000 & 0,2141 & $*$ & 30,12 \\
\hline Escenario 5 & $\begin{array}{c}\text { S42 } \\
\text { S420 } \\
\text { S211 } \\
\text { S110 } \\
\text { S323 } \\
\text { S510 } \\
\text { S321 }\end{array}$ & $\begin{array}{l}3,1400 \\
3,3750 \\
3,4510 \\
3,4803 \\
3,5034 \\
3,5828 \\
3,7347\end{array}$ & 0,0001 & & 0,0001 & 0,15634 & $\begin{array}{c}\text { S211-S321* } \\
\text { S211-S42* } \\
\text { S420-S321* } \\
\text { S420-S42* } \\
\text { S110-S321* } \\
\text { S110-S42* } \\
\text { S323-S42* } \\
\text { S321-S42* } \\
\text { S510-S42* }\end{array}$ & $\begin{array}{l}10,37 \\
14,53 \\
15,14 \\
10,98 \\
10,26 \\
15,90 \\
16,98 \\
27,79 \\
20,69\end{array}$ \\
\hline
\end{tabular}

(1) Se ha utilizado el test de Wilcoxon para comparar las medianas de 2 fotografías y el test de Test Kruskal Walis cuando en el escenario había más de dos fotos.

* Indica diferencias significativas.

En los escenarios 2 y 4 se esperaba que la aplicación de plantaciones de árboles en la boca del túnel diera como resultado un incremento en la calidad paisajística de la zona. Sin embargo en el caso del escenario 2 se ha obtenido una disminución de la calidad del paisaje del 19,18\%, al introducir un denso bosquete en una foto original de taludes hidrosembrados próximos a la boca del túnel. Por otro lado, la inclusión de una pequeña plantación a la entrada del túnel en el escenario 3 ha dado como resultado un incremento de tan solo un $5 \%$, el cual no es estadísticamente significativo con un nivel de confianza del 5\%.

En el escenario 5 se evaluaron diferentes acabados para el emboquillado de un túnel. En la Tabla 6 solo se muestran los 9 pares de fotografías en los que el test confirma diferencias significativas entre las medias. Este análisis muestra que existen diferencias entre el túnel mejor valorado (S321), terminado en pico de flauta, y los acabados en marco artístico (S211), en encachado (S420), en marco en ladrillo (S110) y como peor valorado el terminado en placas prefabricadas (S42), Ilegando a diferenciarse su valoración en un $28 \%$. Además, cabe destacar el hecho de que el peor valorado (S42) muestra diferencias significativas con todos los demás.

Las bocas de túnel con una mayor preferencia del público son las que intentan integrar el túnel con el paisaje, reduciendo la alteración que éste produce en el mismo. Dentro de este grupo destacan las bocas con pico de flauta, seguidas de las bocas más frecuentes en nuestras carreteras, las bocas circulares o rectangulares con borde fino. En cambio, en las bocas de túnel donde se destaca la presencia del mismo con la inclusión de emboquillados con marcos artísticos, encachados o muros prefabricados, la valoración disminuye.

\subsubsection{Pantallas vegetales}

En la Tabla 7 se muestran los resultados de los test estadísticos empleados para identificar las diferencias significativas entre las valoraciones realizadas a los escenarios 1, 2 y 3 de pantallas vegetales. En todos los casos se encontraron diferencias significativas. 
Tabla 7. Resultados en las fotografías con pantallas vegetales

\begin{tabular}{|c|c|c|c|c|c|c|c|c|}
\hline Escenario & Fotos & media & $\begin{array}{l}\text { ADEVA } \\
\text { p-valor }\end{array}$ & $\begin{array}{c}\text { Test T Student } \\
\text { p-valor }\end{array}$ & $\begin{array}{c}\text { Test Wilcoxon } \\
\text { p-valor }\end{array}$ & $\begin{array}{l}\text { Test Cochran } \\
\text { p-valor }\end{array}$ & Test múltiples rangos & $\begin{array}{c}\text { Diferencia } \\
\text { entre medias } \\
(\%)\end{array}$ \\
\hline Escenario 1 & $\begin{array}{l}\text { S325 } \\
\text { S112 } \\
\end{array}$ & $\begin{array}{l}3,0272 \\
3,5855 \\
\end{array}$ & 0 & 0 & 0 & 0,9457 & $*$ & 27,54 \\
\hline Escenario 2 & $\begin{array}{c}\mathrm{S} 118 \\
\text { S29 } \\
\end{array}$ & $\begin{array}{c}2,7171 \\
3,085 \\
\end{array}$ & 0,0011 & 0 & 0,0001 & 0,0495 & $*$ & 21,43 \\
\hline Escenario 3 & $\begin{array}{l}\text { S410 } \\
\text { S513 }\end{array}$ & $\begin{array}{l}1,9474 \\
2,5629\end{array}$ & 3 & 0 & 0 & 0,7106 & $*$ & 64,97 \\
\hline
\end{tabular}

* Indica diferencias significativas.

Los resultados obtenidos muestran la efectividad conseguida en la integración paisajística al introducir una pantalla vegetal con la intención de ocultar una alteración visual del paisaje al observador. La aplicación de una misma medida obtiene una mejoría notable en los escenarios 1 y 2 $(27,54 \%$ y $21,43 \%)$, y un $64,97 \%$ de aumento en la calidad final del paisaje en el caso del escenario 3. Es interesante recalcar que, pese a que los escenarios 1 y 2 poseen mayor valoración de partida, es en el escenario 3, con peor valoración de partida (S410), donde se obtiene el mayor aumento al introducir las pantallas vegetales.

\subsubsection{Taludes}

En este caso, a la vista de los resultados presentados en la Tabla 8, los tres escenarios muestran diferencias significativas en cuanto a su valoración.
El escenario donde se ha experimentado una mayor mejoría ha correspondido con la colocación de una manta orgánica y posterior hidrosembrado sobre un talud rocoso, obteniéndose una mejora del 50,77\%. En cambio, soluciones donde se ha incorporado mayor riqueza florística con árboles y arbustos han obtenido mejoras menos destacadas (35,92 y 31,40\% respectivamente). Este resultado podría indicar que la presencia de cortes en la roca, cuyo aspecto puede parecer muy artificial, supone un gran impacto paisajístico.

\subsubsection{Pasos superiores}

Tanto el escenario 1 como el escenario 3, donde se comparan dos fotos, las diferencias son significativas (Tabla 9). En el escenario 2 (Tabla 9), se forman dos grupos en los que las fotos no se diferencian entre ellas, pero sí con las del grupo al que no pertenecen.

Tabla 8. Resultados en las fotografías con taludes

\begin{tabular}{|c|c|c|c|c|c|c|c|c|}
\hline Escenario & Fotos & media & $\begin{array}{l}\text { ADEVA } \\
\text { p-valor }\end{array}$ & $\begin{array}{l}\text { Test T Student } \\
\text { p-valor }\end{array}$ & $\begin{array}{l}\text { Test Medianas(1) } \\
\text { p-valor }\end{array}$ & $\begin{array}{l}\text { Test Cochran } \\
\text { p-valor }\end{array}$ & Test múltiples rangos & $\begin{array}{c}\text { Diferencia } \\
\text { entre medias } \\
(\%)\end{array}$ \\
\hline Escenario 1 & $\begin{array}{l}\text { S111 } \\
\text { S220 }\end{array}$ & $\begin{array}{l}2,5592 \\
3,1192\end{array}$ & 0,0000 & 0,0000 & 0,0000 & 0,7182 & * & 35,92 \\
\hline Escenario 2 & $\begin{array}{l}\text { S55 } \\
\text { S28 }\end{array}$ & $\begin{array}{l}2,5563 \\
3,3464\end{array}$ & 0,0000 & 0,0000 & 0.0000 & 0,7681 & * & 50,77 \\
\hline Escenario 3 & $\begin{array}{l}\text { S315 } \\
\text { S116 } \\
\text { S417 }\end{array}$ & $\begin{array}{l}2,55705 \\
2,78289 \\
3,04605\end{array}$ & 0,0001 & & 0.0001 & 0,2968 & $\begin{array}{l}\text { S315-S116* } \\
\text { S315-S417* } \\
\text { S116-S417* }\end{array}$ & $\begin{array}{l}14,50 \\
31,40 \\
14,76\end{array}$ \\
\hline
\end{tabular}

(1) Se ha utilizado el test de Wilcoxon para comparar las medianas de 2 fotografías y el test de Test Kruskal Walis cuando en el escenario había más de dos fotos. * Indica diferencia significativa.

Tabla 9. Resultados en las fotografías con pasos superiores

\begin{tabular}{|c|c|c|c|c|c|c|c|c|}
\hline Escenario & Fotos & media & $\begin{array}{l}\text { ADEVA } \\
\text { p-valor }\end{array}$ & $\begin{array}{l}\text { Test T Student } \\
\text { p-valor }\end{array}$ & $\begin{array}{c}\text { Test Medianas } \\
\text { p-valor }\end{array}$ & $\begin{array}{l}\text { Test Cochran } \\
\text { p-valor }\end{array}$ & Test múltiples rangos & $\begin{array}{c}\text { Diferencia } \\
\text { entre medias } \\
(\%)\end{array}$ \\
\hline Escenario 1 & $\begin{array}{l}\text { S412 } \\
\text { S114 }\end{array}$ & $\begin{array}{l}2,6579 \\
3,1184\end{array}$ & 0,0000 & 0,0000 & 0,0000 & 0,7182 & $*$ & 27,28 \\
\hline Escenario 2 & $\begin{array}{c}\text { S126 } \\
\text { S415 } \\
\text { S33 } \\
\text { S224 } \\
\end{array}$ & $\begin{array}{c}2,2 \\
2,32667 \\
2,91781 \\
2,91892 \\
\end{array}$ & 0.000 & & 0.000 & 0,675499 & $\begin{array}{c}\text { S126-S224* } \\
\text { S126-S33* } \\
\text { S224-S415* } \\
\text { S415-S33* }\end{array}$ & $\begin{array}{l}59,91 \\
59,82 \\
44,64 \\
44,56 \\
\end{array}$ \\
\hline Escenario 3 & $\begin{array}{c}\text { S518 } \\
\text { S37 } \\
\end{array}$ & $\begin{array}{l}2,9600 \\
3,3311 \\
\end{array}$ & 0,0010 & 0,0005 & 0,0000 & 0,7681 & * & 18,93 \\
\hline Escenario 4 & $\begin{array}{l}\text { S411 } \\
\text { S524 } \\
\text { S517 } \\
\text { S320 } \\
\text { S12 } \\
\text { S212 } \\
\text { S222 }\end{array}$ & $\begin{array}{c}2,47368 \\
2,5473 \\
2,57333 \\
2,66216 \\
2,75817 \\
2,84314 \\
2,8543\end{array}$ & 0,0033 & & 0.0000 & 0,0910 & $\begin{array}{l}\text { S222-S411* } \\
\text { S222-S517* } \\
\text { S222-S524* } \\
\text { S12-S411* } \\
\text { S411-S212* } \\
\text { S517-S212* } \\
\text { S524-S212* }\end{array}$ & $\begin{array}{l}25,83 \\
17,86 \\
19,84 \\
19,30 \\
25,83 \\
17,15 \\
19,12\end{array}$ \\
\hline
\end{tabular}

(1) Se ha utilizado el test de Wilcoxon para comparar las medianas de 2 fotografías y el test de Test Kruskal Walis cuando en el escenario había más de dos fotos. * Indica diferencia significativa. 
El primer grupo, que obtiene una valoración más baja, es el formado por las fotos $\$ 126$ y S415, en los que se han introducido cambios de color en la barandilla, pero no de vegetación. El otro grupo, con mejor valoración, lo forman las fotos en las que la vegetación se ha incrementado en densidad y diversidad de especies, es decir, las fotos S33 y S224.

En el caso del escenario 4, el test de los múltiples rangos señala diferencias significativas en 7 pares de fotografías. La foto del paso superior sin pilar central, con estribos pequeños y viga gruesa (S222), obtiene una valoración significativamente mejor que las obtenidas por las fotografías S411, S517 y S524. Estas tres fotografías son las peor valoradas y tienen en común la presencia de un pilar central. El segundo mejor valorado (S212) es significativamente mejor que los 3 peores valorados, en cambio, el tercer mejor valorado (S12) tan solo es significativamente mejor que el peor valorado.

\subsubsection{Muros de contención de tierras}

En el escenario estudiado, tan solo existen diferencias significativas entre el muro peor valorado (muro de piezas prefabricadas S43) y el resto de las fotografías, donde los muros están construidos con materiales o acabados más parecidos a la naturaleza (ver Tabla 10).
En el estudio de las soluciones constructivas de muros de contención de tierras se han obtenido valores muy similares de preferencia entre los encuestados. Los resultados obtenidos han coincidido parcialmente con la hipótesis de partida fijada. La utilización de materiales que se asemejan a la naturaleza ha obtenido mejores valoraciones. Un ejemplo de esto es la solución de muro con jardineras (S24) o el muro con acabado en cara rústica (S14).

\subsubsection{Pantallas acústicas}

En los escenarios 1 y 3 se comprueba que las diferencias son significativas mediante la ocultación de la pantalla prefabricada con una pantalla vegetal o la sustitución de una pantaIla sólida por una transparente (Tabla 11).

En el caso del escenario 2 (Tabla 11), donde la pantalla acústica opaca se sustituye por dos soluciones diferentes, las diferencias son significativas entre la pantalla original y las dos introducidas (S49 es diferente de S312 y S520), pero no existe una diferencia destacable entre las dos alternativas propuestas (no existen diferencias significativas entre S520 y S312).

Las mejoras más destacadas se obtienen cuando se consigue reducir la percepción de la pantalla en el paisaje, obteniéndose así una mejora en torno al 45,71 \% (S27 y S117).

Tabla 10. Resultados en las fotografías con muros de contención de tierras

\begin{tabular}{lccccccc}
\hline Escenario & Fotos & media & $\begin{array}{c}\text { ADEVA } \\
\text { p-valor }\end{array}$ & $\begin{array}{c}\text { Test Kruskal Wallis } \\
\text { p-valor }\end{array}$ & $\begin{array}{c}\text { Test Cochran } \\
\text { p-valor }\end{array}$ & Test múltiples rangos & $\begin{array}{c}\text { Diferencia } \\
\text { entre medias } \\
(\%)\end{array}$ \\
\hline & S43 & 2,81046 & & & & S322-S43* & 28,88 \\
Escenario 1 & S58 & 3,17219 & & & & S58-S43* & 19,98 \\
& S14 & 3,33333 & 0,0000 & 0,0000 & 0,4515 & S24-S43* & 32,49 \\
& S24 & 3,34868 & & & S43-S14* & 29,73 \\
\hline
\end{tabular}

* Indica diferencias significativas

Tabla 11. Resultados en las fotografías con pantallas acústicas

\begin{tabular}{|c|c|c|c|c|c|c|c|c|}
\hline Escenario & Fotos & media & $\begin{array}{l}\text { ADEVA } \\
\text { p-valor }\end{array}$ & $\begin{array}{c}\text { Test T Student } \\
\text { p-valor }\end{array}$ & $\begin{array}{c}\text { Test medianas } \\
\text { p-valor }\end{array}$ & $\begin{array}{c}\text { Test Cochran } \\
\text { p-valor }\end{array}$ & Test múltiples rangos & $\begin{array}{c}\text { Diferencia } \\
\text { entre medias } \\
(\%)\end{array}$ \\
\hline Escenario 1 & $\begin{array}{l}\text { S117 } \\
\text { S27 } \\
\end{array}$ & $\begin{array}{l}2,6776 \\
3,4444 \\
\end{array}$ & 0,000 & 0,000 & 0,000 & 0,5791 & $*$ & 45,71 \\
\hline Escenario 2 & $\begin{array}{c}\mathrm{S} 49 \\
\mathrm{~S} 312 \\
\mathrm{~S} 520 \\
\end{array}$ & $\begin{array}{l}2,81333 \\
3,08219 \\
3,14865 \\
\end{array}$ & 0,0013 & & 0,0008 & 0,416652 & $\begin{array}{l}\text { S520-S49* } \\
\text { S312-S49* }\end{array}$ & $\begin{array}{l}18,49 \\
14,83\end{array}$ \\
\hline Escenario 3 & $\begin{array}{l}\text { S515 } \\
\text { S317 }\end{array}$ & $\begin{array}{l}2,6600 \\
3,2617\end{array}$ & 0,000 & 0,000 & 0,000 & 0,6536 & $*$ & 36,25 \\
\hline
\end{tabular}

(1) Se ha utilizado el test de Wilcoxon para comparar las medianas de 2 fotografías y el test de Test Kruskal Walis cuando en el escenario había más de dos fotos. * Indica diferencia significativa.

Tabla 12. Análisis de las diferencias significativas en las fotografías con medianas

\begin{tabular}{|c|c|c|c|c|c|c|c|c|}
\hline Escenario & Fotos & media & $\begin{array}{l}\text { ADEVA } \\
\text { p-valor }\end{array}$ & $\begin{array}{l}\text { Test T Student } \\
\text { p-valor }\end{array}$ & $\begin{array}{c}\text { Test Wilcoxon } \\
\text { p-valor }\end{array}$ & $\begin{array}{c}\text { Test Cochran } \\
\text { p-valor }\end{array}$ & Test múltiples rangos & $\begin{array}{c}\text { Diferencia } \\
\text { entre medias } \\
(\%)\end{array}$ \\
\hline Escenario 1 & $\begin{array}{l}\text { S215 } \\
\text { S120 }\end{array}$ & $\begin{array}{l}3,0197 \\
3,4803\end{array}$ & 0,000 & 0,000 & 0,000 & 0,3246 & * & 22,81 \\
\hline Escenario 2 & $\begin{array}{l}\text { S52 } \\
\text { S122 }\end{array}$ & $\begin{array}{l}3,0265 \\
3,2566\end{array}$ & 0,0404 & 0,020 & 0,0073 & 0,6011 & $*$ & 11,35 \\
\hline Escenario 3 & $\begin{array}{l}\text { S46 } \\
\text { S34 }\end{array}$ & $\begin{array}{l}3,3856 \\
3,5828\end{array}$ & 0,0654 & 0,030 & 0,0697 & 0,0180 & & \\
\hline
\end{tabular}


En el caso del escenario 2, las mejoras introducidas consiguen una mejora de menor importancia, próximas al 19 \% (S312 y S520).

\subsubsection{Medianas}

En este caso, las mejoras introducidas en los escenarios 1 y 2 dan como resultado mejoras significativas en la percepción. Sin embargo, en el escenario 3, el revegetado de la mediana no dio lugar a cambios (Tabla 12).

Los resultados obtenidos en los escenarios estudiados han sido poco Ilamativos. Se ha obtenido una mejora del 22,81\% para el escenario 1 y del $11,35 \%$ en el escenario 2, en cambio en el escenario 3, con mayor calidad de paisaje, las diferencias han resultado no ser significativas.

\section{DISCUSIÓN}

En este trabajo se han identificado las medidas de integración paisajística recomendadas por guías y manuales y se ha cuantificado en qué grado las medidas de integración propuestas pueden variar la percepción del paisaje por el observador/usuario. Así, se han extraído e identificado los esfuerzos de integración que obtienen mejores resultados, según la percepción de observadores/ usuarios, en paisajes donde están presentes elementos específicos de las infraestructuras como emboquillados de túneles, pasos superiores, pantallas vegetales, taludes, muros de contención, pantallas acústicas o medianas. Estos resultados pueden servir de base para la elaboración de guías y manuales, orientando las buenas prácticas de integración paisajística en la fase de planificación, realización de proyectos y gestión de concesionarias de autopistas.

A continuación se discuten los resultados obtenidos en los diferentes elementos de las autopistas estudiados:

\subsection{La integración paisajística de túneles}

Según la literatura consultada, los diseños deben intentar reducir al máximo el impacto visual de la presencia del túnel en la montaña. Por ello, las soluciones que se asemejen a elementos presentes en la naturaleza, como sería la entrada redondeada de una cueva, serían los preferidos entre los observadores $(23,7)$. Los resultados obtenidos muestran que el público prefiere bocas de túnel redondeadas frente a cuadradas o llamativas.

También es necesario recuperar la zona que rodea el emboquillado (23), sin embargo, la vegetación demasiado abundante o frondosa no ha dado buenos resultados en este estudio. Sí se han detectados mejoras en las preferencias expresadas por el público al usar colores miméticos con el paisaje donde esté localizado el túnel (23).

\subsection{Integración paisajística de pantallas vegetales}

Teniendo en cuenta los resultados obtenidos en los escenarios relacionados con pantaIlas vegetales, los encuestados han valorado mejor las fotografías donde se habían ocultado alteraciones en el paisaje mediante vegetación. Estas mejoras (21-65\%) se producen con la ocultación de elementos artificiales y son compatibles con los resultados de estudios previos consultados $(18,24)$.

\subsection{Integración paisajística de taludes}

En los escenarios evaluados se han realizado modificaciones al incluir plantaciones de arbolado, matorrales y mantas orgánicas con posterior hidrosembrado, estas intervenciones paisajísticas se recomiendan en la literatura de paisajismo de carreteras (8, 25, 26, 27). Analizando por separado la aplicación de cada una de las medidas, se han obtenido mejoras importantes en todas ellas, con rangos de mejora entre el 30 y el $50 \%$.

La mejora conseguida en el paisaje con la integración de un talud estará condicionada por las características del paisaje en el que se encuentra y su calidad. No siempre un talud con árboles es el talud mejor integrado. En ocasiones, una integración con matorral o hidrosembrado puede estar mejor integrado. Además, a la vista de la gran mejora que se obtiene en el escenario 2, debe tenerse en cuenta que la aparición de un talud rocoso puede introducir una alteración mayor en el paisaje que otro tipo de taludes.

\subsection{Integración paisajística de pasos superiores}

Las recomendaciones de diseño de pasos superiores invitan a utilizar formas y colores compatibles con el medio que los rodea, a buscar ligereza en los elementos que conforman la estructura y a revegetar los taludes laterales que se generan tras la construcción de estos pasos (7, 23, 24, 26).

El uso de colores miméticos o neutros en elementos metálicos del paso superior es recomendable. Parece especialmente efectivo si los taludes del paso superior carecen de vegetación, ya que la incorrecta elección de colores se hace más Ilamativa.

Los resultados muestran que las plantaciones en los taludes del paso superior mejoran de forma destacada la integración del mismo en el paisaje de las fotos utilizadas. 
Esta integración es mayor si existe presencia de árboles en las inmediaciones.

El diseño constructivo del paso superior influye en la percepción de los observadores. Las formas curvas y los diseños más transparentes son preferidos por los usuarios. A la hora de diseñar con estos criterios, se debe encontrar el equilibrio entre transparencia y ligereza con la sensación de robustez y seguridad del mismo.

\subsection{Integración paisajística de muros de contención de tierras}

Numerosos manuales y guías de integración paisajística recomiendan el uso de materiales con apariencia natural (8), colores miméticos con el paisaje y la incorporación de patrones y texturas en el acabado del muro de contención, todo ello orientado a reducir la alteración introducida por éstos en la percepción del paisaje interior de la carretera $(23,26)$.

Según los resultados obtenidos, se ha confirmado una mejora generalizada en aquellos diseños que se alejaban de las formas rectas y paramentos simples de los muros de placas de hormigón. Las mejoras obtenidas han sido de entre el 20 y el $30 \%$.

La utilización de muros de escamas prefabricadas de hormigón puede ser una solución constructiva alternativa muy interesante desde el punto de vista paisajístico, puesto que es la solución mejor valorada entre las dispuestas en la encuesta.

En futuras investigaciones se debería estudiar en mayor profundidad la integración paisajística de los muros de gaviones con diferentes materiales de relleno y con diferentes tipologías de paisajes (especialmente paisajes poco vegetados de la costa mediterránea). A tenor de los resultados obtenidos, el nivel de naturalidad conseguida con esta solución constructiva podría cuestionarse.

Las soluciones constructivas más valoradas (muro con acabado rústico, jardineras y de escamas) han obtenido una valoración similar entre los encuestados, por tanto, podría ser interesante estimar sus costes de construcción con la intención de completar el estudio.

\subsection{Integración paisajística de pantallas acústicas}

En relación con una mejor integración de pantallas acústicas en el paisaje, se ha comprobado que las recomendaciones de manuales y guías han coincidido con la opinión de la mayor parte de los encuestados.
La bibliografía recomienda la ocultación de pantallas acústicas opacas mediante la creación de pantallas vegetales $(26,27)$. Este tipo de intervención ha obtenido una buena acogida por el público encuestado. Asimismo, la sustitución de pantallas opacas de colores miméticos por pantallas transparentes también ha obtenido buenos resultados $(7,24,28)$. Cabe destacar que en ambas medidas se obtiene una mejoría con valores superiores al $30 \%$.

\subsection{Integración paisajística de medianas}

Las plantaciones en medianas tienen una gran influencia en la seguridad de las autopistas, puesto que dirigen la vista y evitan deslumbramientos $(7,27)$ y, además, de mejoran la calidad del paisaje interior de la carretera. Pero éstas tienen necesidades especiales de mantenimiento, dado su difícil acceso para los operarios. Según la encuesta, la presencia de medianas estrechas con plantaciones de adelfas, las cuales deben ser recortadas de manera frecuente (escenario 3), no han sido valoradas mejor por parte del público. Cabe destacar el importante sobrecoste que llevan asociados estos recortes, lo cual pone en entredicho su justificación por criterios estéticos. Como excepción se ha obtenido que la incorporación de especies vegetales con flor es un recurso interesante en las medianas, especialmente en aquellos paisajes con calidades estéticas menores.

\section{CONCLUSIONES}

Una conclusión importante de este estudio es que la "escena interior" de la carretera es relevante a la hora de evaluar el paisaje observado desde la carretera $y$, por tanto, debe ser incluida tanto en los modelos de definición de soluciones de integración paisajística de la carretera como a la hora de evaluar alternativas de trazado o proyecto.

Además, se pueden extraer las siguientes conclusiones particulares en función de tipo de medida de integración estudiada:

- En túneles, los diseños de emboquillados redondeados, con formas que dan sensación de dinamismo y con colores miméticos son preferibles.

- Las ocultaciones de alteraciones visuales mediante pantallas vegetales consiguen mejorar la calidad del paisaje.

- En el tratamiento de los taludes debe tenerse en cuenta las características del paisaje del entorno. Debe prestarse especial atención a los taludes en roca.

- En pasos superiores se deben tener en cuenta tanto la estructura, materiales y acabados, como la revegetación de los estribos. Las formas transparentes y los 
taludes revegetados mejoran la percepción del público.

- Los diseños de muros cuyos acabados se alejan de formas rectas y paramentos simples son mejor valorados.

- Las pantallas acústicas opacas introducen una alteración en el paisaje, aunque puede reducirse con materiales transpa- rentes o con pantallas vegetales delante de las mismas.

- Revegetar medianas no siempre consigue mejorar la calidad del paisaje. Es interesante estudiar en más profundidad las medianas recortadas con formas artificiales dado su mayor coste de conservación.

\section{BIBLIOGRAFÍA}

(1) Nogué, J.; Salas, P.: "Conceptual, methodological and procedural bases for the preparation of the Catalan Landscape Catalogues". Obsservatori del Paitsaje. Olot and Barcelona (2006). http:// www.catpaisatge.net/fitxers/SummaryPrototype.pdf

(2) Otero, I. et. al.: "La carretera como elemento de valor paisajístico y medioambiental. Captación del valor del paisaje a través de la carretera". Informes de la Construcción, vol. 58, n. ${ }^{o} 504$ (2006), pp. 39-54

(3) Glaría, G.; Ceñal, M. ${ }^{\circ}$ A.: "Impactos paisajísticos de carreteras y medidas correctoras. Niveles de detaIle". Informes de la Construcción. Monográfico Carretera y Medioambiente, vol. 44, n. ${ }^{\circ}$ 425-426 (1993).

(4) Potter, R. D; Wagar, J. A.: "Techniques for Inventoring Manmade Impacts in Roadway Enviroments". Pacific Northwest Forest and Range Experiment Station Forest Service, U.S. Department of Agriculture (1971). Portland. Oregon.

(5) Orea, D.; Villarino, T.: "Experiencia en la evaluación de impacto ambiental de carreteras". Informes

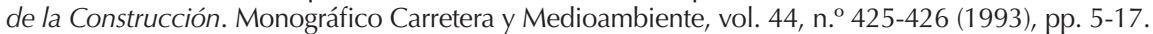

(6) Loro, M.; Martín, B.; Otero, I.; Arce, R.; Cañas, I.: "Análisis de las medidas de integración paisajística utilizadas en las autopistas españolas". 7-9 Julio. Congreso de Ingeniería del transporte. Madrid, 2010.

(7) Junta de Andalucía. La carretera en el paisaje. Criterios para su planificación trazado y proyecto. p. 244, Consejería de Obras públicas y transportes. Centro de Estudios de paisaje y Territorio. Sevilla, 2009.

(8) Rubio, S.; Días, J.; Laorden, L.; Rodrigo, M.; Zaragoza, A.: La carretera en la sociedad del siglo XXI. Respuestas a nuevos retos. Asociación Española de la Carretera (AEC). Madrid, 2006. http://www. aecarretera.com/Libro\%20definitivo.pdf.

(9) Comunidad Valenciana. Decreto 120/2006, de 11 de agosto, del Consell, por el que se aprueba el Reglamento del Paisaje de la Comunidad Valenciana. DOGV, n. ${ }^{\circ}$ 5325, de 16 de agosto de 2006.

(10) Ayuntamiento de Valencia. Contenido obligatorio de los Estudios de Paisaje del PGOU de Valencia. Normas de Integración Paisajística. DOGV, n. ${ }^{\circ} 2015$, de 3 de mayo de 1993.

(11) Fernández, S.: "Participación pública, gobierno del territorio y paisaje en la Comunidad de Madrid". Boletín de la Asociación de Geógrafos Españoles (A.G.E.). n. ${ }^{\circ} 46$ (2008).

(12) Kaplan, R.: "The Analysis of Perception via Preference: A Strategy for Studying How the Environment Is Experienced". Landscape and Urban Planning. Vol. 12, (1985), pp. 161-176.

(13) Sullivan, W. C.: "Perceptions of the Rural-Urban Fringe: Citizen Preferences for Natural and Developed Settings". Landscape and Urban Planning. Vol. 29 (1994), pp. 85-101.

(14) Stamps, A. E.: "Use of Photographs to Simulate Environments: A Meta-Analysis". Perceptual and Motor Skills. Vol. 71 (1990), pp. 907-913

(15) Cañas, I.; Ayuga, E.; Ayuga, F.: "A contribution to the assessment of scenic quality of landscapes based on preferences expressed by the public". Land Use Policy. Vol. 26 (2009), pp. 1173-1181.

(16) Wolf, K. L.: "Assessing public response to freeway roadsides. Urban Forestry and context-sensitive solutions". Journal of the Transportation Research Board. n. ${ }^{\circ} 1984$ (2006), pp. 102-111.

(17) Wolf, K. L.: "Community Context and Strip Mall Retail. Public Response to the Roadside Landscape". Journal of the Transportation Research Board. $\mathrm{n}^{\circ} 2060$ (2008), pp.95-103.

(18) Garré, S.; Meeus, S.; Gulink, H.: "The dual role of roads in the visual landscape: A case-study in the area around Mechelen (Belgium)". Landscape and Urban Planning. Vol. 92 (2009), pp. 125-135.

(19) Cañas, I.: Introducción al Paisaje. Unicopia. Madrid, 1995.

(20) Otero, I.; Ortega, E.: "Evaluation of landscape around roads". En $2^{\text {nd }}$ conf. Environment \& Transport, incl. $15^{\text {th }}$ conf. Transport and Air Pollution. Reims, France. Proceedings, Actes Inrets, n. ${ }^{\circ} 107$, vol. 2, Arcueil, France, 2006.

(21) Cañas, I.: "Estimación del impacto paisajístico de las carreteras". Informes de la Construcción. Monográfico Carretera y Medioambiente, vol. 44, n. ${ }^{\circ} 425-426$ (1993), pp. 43-53.

(22) Otero, I.; Casermeiro, M. A.; Ezquerra, A.; Esparcia, P.: "Landscape evaluation: comparison of evaluation methods in a region of Spain". Journal of Environmental Management, vol. 85 (2007), pp. 204-214.

(23) GISA, Libro de Estilo de las Carreteras Catalanas. Generalidad de Cataluña, Departamento de Política Territorial y Obras Públicas. 295 pp. PASA A 25. Barcelona, 2009.

(24) The Highways Agency, Scotish Executive, Welsh Assembly Government and the Department for Regional Development Northern Ireland. Design Manual for Roads and Bridges. Vol. 10 Environmental design and management section 3: Landscape management. 2004. http://www.standardsforhighways.co.uk/dmrb/.

(25) Ministerio de Fomento y Esteyco. Guía Metodológica para la inclusión de proyectos ambientales en los proyectos de carreteras. Dirección General de Carreteras, Subdirección General de proyectos. Madrid, 1999.

(26) Texas Department of Transportation. Landscape and Aesthetics design manual. 2009.

(27) Transit New Zealand. Guidelines for Highway Landscaping. NZTransport Agency. New Zealand, 2006.

(28) Segués, F.; Alegre, D.: "Gestión del ruido en infraestructuras de transporte. Metodología para elaboración de mapas de ruido y técnicas de reducción de los niveles sonoros mediante pavimentos absorbentes y sistemas de apantallamiento". Carreteras, extraordinario ambiental 2006. 